Article

\title{
On Some Sufficient Conditions for a Function to Be $p$-Valent Starlike
}

\author{
Mamoru Nunokawa ${ }^{1}$, Janusz Sokół ${ }^{2, *(D)}$ and Edyta Trybucka ${ }^{2}$ (D) \\ 1 Department of Mathematics, University of Gunma, Hoshikuki-cho 798-8, Chuou-Ward, \\ Chiba 260-0808, Japan; mamoru_nuno@doctor.nifty.jp \\ 2 College of Natural Sciences, University of Rzeszów, ul. Prof. Pigonia 1, 35-310 Rzeszów, Poland; \\ eles@ur.edu.pl \\ * Correspondence: jsokol@ur.edu.pl
}

Received: 21 October 2019; Accepted:10 November 2019; Published: 15 November 2019

\begin{abstract}
A function $f$ analytic in a domain $D \in \mathbb{C}$ is called $p$-valent in $D$, if for every complex number $w$, the equation $f(z)=w$ has at most $p$ roots in $D$, so that there exists a complex number $w_{0}$ such that the equation $f(z)=w_{0}$ has exactly $p$ roots in $D$. The aim of this paper is to establish some sufficient conditions for a function analytic in the unit disc $\mathbb{D}$ to be $p$-valent starlike in $\mathbb{D}$ or to be at most $p$-valent in $\mathbb{D}$. Our results are proved mainly by applying Nunokawa's lemmas.
\end{abstract}

Keywords: univalent functions; starlike; convex; close-to-conve

MSC: primary 30C45; secondary 30C 80

\section{Introduction}

A function $f$ analytic in a domain $D \in \mathbb{C}$ is called $p$-valent in $D$, if for every complex number $w$, the equation $f(z)=w$ has at most $p$ roots in $D$, so that there exists a complex number $w_{0}$ such that the equation $f(z)=w_{0}$ has exactly $p$ roots in $D$. We denote by $\mathcal{H}$ the class of functions $f$ which are holomorphic in the open unit unit $\mathbb{D}=\{z \in \mathbb{C}:|z|<1\}$. Denote by $\mathcal{A}_{p}, p \in \mathbb{N}=\{1,2, \ldots\}$, the class of functions $f \in \mathcal{H}$ given by

$$
f(z)=z^{p}+\sum_{n=p+1}^{\infty} a_{n} z^{n}, \quad z \in \mathbb{D} .
$$

Let $\mathcal{A}=\mathcal{A}_{1}$. The well known Noshiro-Warschawski univalence condition, (see [1,2]) indicates that if $f$ is analytic in a convex domain $D \subset \mathbb{C}$ and

$$
\mathfrak{R e}\left\{e^{i \theta} f^{\prime}(z)\right\}>0, \quad z \in D,
$$

for some real $\theta$, then $f$ is univalent in $D$. In [3] Ozaki extended the above result by showing that if $f$ of the form (1) is analytic in a convex domain $D$ and for some real $\theta$ we have

$$
\mathfrak{R e}\left\{e^{i \theta} f^{(p)}(z)\right\}>0, \quad z \in D,
$$

then $f$ is at most $p$-valent in $D$. In [4] it was proved that if $f \in \mathcal{A}_{p}, p \geq 2$, and

$$
\left|\arg \left\{f^{(p)}(z)\right\}\right|<\frac{3 \pi}{4}, \quad z \in \mathbb{D},
$$

then $f$ is at most $p$-valent in $\mathbb{D}$. 
If $f \in \mathcal{H}$ satisfies $f(0)=0, f^{\prime}(0)=1$ and

$$
\mathfrak{R e}\left\{\frac{z f^{\prime}(z)}{f(z)}\right\}>0, \quad z \in \mathbb{D},
$$

then $f$ is said to be starlike with respect to the origin in $\mathbb{D}$ and it is denoted by $f \in \mathcal{S}^{*}$. It is known that $\mathcal{S}^{*} \subset \mathcal{S}$, where $\mathcal{S}$ denotes the class of all functions in $\mathcal{A}$ which are univalent in $\mathbb{D}$. Moreover, let $\mathcal{S}_{p}^{*}$ and $\mathcal{C}_{p}$ be the subclasses of $\mathcal{A}_{p}$ defined as follows

$$
\begin{aligned}
& \mathcal{S}_{p}^{*}=\left\{f \in \mathcal{A}_{p}: \mathfrak{R e}\left\{\frac{z f^{\prime}(z)}{f(z)}\right\}>0, z \in \mathbb{D}\right\}, \\
& \mathcal{C}_{p}=\left\{f \in \mathcal{A}_{p}: z f^{\prime}(z) / p \in \mathcal{S}_{p}^{*}\right\} .
\end{aligned}
$$

$\mathcal{S}_{p}^{*}$ is called the class of $p$-valent starlike functions and $\mathcal{C}_{p}$ is called the class of $p$-valent convex functions. Note that $\mathcal{S}_{1}^{*}=\mathcal{S}^{*}$ and $\mathcal{C}_{1}=\mathcal{C}$, where $\mathcal{S}^{*}$ and $\mathcal{C}$ are usual classes of starlike and convex functions, respectively. A function $f \in \mathcal{A}_{p}$ is said to be an element of the class $\mathcal{K}_{p}$ of $p$-valent close-to-convex functions if there exists a function $g \in \mathcal{C}_{p}$ for which

$$
\mathfrak{R e}\left\{\frac{f^{\prime}(z)}{g^{\prime}(z)}\right\}>0, \quad z \in \mathbb{D} .
$$

In [5] (Th.1) Umezawa proved the following theorem.

Theorem 1. If $f \in \mathcal{K}_{p}$, then $f$ is at most $p$-valent in $\mathbb{D}$.

Because $\mathcal{C}_{p} \subset \mathcal{S}_{p}^{*} \subset \mathcal{K}_{p}$, we have from Theorem 1 that $p$-valent starlike functions and $p$-valent convex functions are at most $p$-valent in $\mathbb{D}$ too.

\section{Preliminaries}

In this paper we need the following lemmas.

Lemma 1 ([6] (Th.5)). If $f \in \mathcal{A}_{p}$, then for all $z \in \mathbb{D}$, we have

$$
\mathfrak{R e}\left\{\frac{z f^{(p)}(z)}{f^{(p-1)}(z)}\right\}>0 \Rightarrow \forall k \in\{1, \ldots, p\}: \quad \mathfrak{R e}\left\{\frac{z f^{(k)}(z)}{f^{(k-1)}(z)}\right\}>0 .
$$

Lemma 2 ([7]). Let $p$ be an analytic function in $|z|<1$, with $p(0)=1$. If there exists a point $z_{0},\left|z_{0}\right|<1$, such that

$$
\mathfrak{R e}\{p(z)\}>0 \text { for }|z|<\left|z_{0}\right|
$$

and

$$
p\left(z_{0}\right)= \pm i a
$$

for some $a>0$, then we have

$$
\frac{z_{0} p^{\prime}\left(z_{0}\right)}{p\left(z_{0}\right)}=\frac{2 i k \arg \left\{p\left(z_{0}\right)\right\}}{\pi},
$$

for some $k \geq\left(a+a^{-1}\right) / 2 \geq 1$.

Corollary 1. Under the assumptions of Lemma 2, we have from (4)

$$
z_{0} p^{\prime}\left(z_{0}\right)=-k a \leq-\frac{1}{2}\left(a+a^{-1}\right) a=-\frac{1}{2}\left(1+\left|p\left(z_{0}\right)\right|^{2}\right) .
$$


Lemma $3([8]$ (p. 200)). Assume that $q(z)$ is univalent in $\mathbb{D}, q(\mathbb{D})$ is a convex set and $F, G$ are analytic in $\mathbb{D}$. If

$$
\frac{F^{\prime}(z)}{G^{\prime}(z)} \prec q(z), \quad z \in \mathbb{D},
$$

where $G$ satisfies $G(0)=F(0)$ and

$$
\mathfrak{R e}\left\{\frac{z G^{\prime}(z)}{G(z)}\right\}>0, \quad z \in \mathbb{D}
$$

then we have

$$
\frac{F(z)}{G(z)} \prec q(z), \quad z \in \mathbb{D} .
$$

Here $\prec$ means the subordination.

Corollary 2. Let $\alpha<1$ be real number. If $f^{(p-1)}(z), g^{(p-1)}(z)$ are analytic in $\mathbb{D}, f^{(p-1)}(0)=g^{(p-1)}(0)$ and

$$
\mathfrak{R e}\left\{\frac{f^{(p)}(z)}{g^{(p)}(z)}\right\}>\alpha, \quad z \in \mathbb{D},
$$

where g satisfies

$$
\mathfrak{R e}\left\{\frac{z g^{(p)}(z)}{g^{(p-1)}(z)}\right\}>0, \quad z \in \mathbb{D}
$$

then we have

$$
\mathfrak{R e}\left\{\frac{f^{(p-1)}(z)}{g^{(p-1)}(z)}\right\}>\alpha, \quad z \in \mathbb{D} .
$$

\section{Main Results}

Theorem 2. Let $f, g \in \mathcal{A}_{p}$. Assume that

$$
\mathfrak{R e}\left\{\frac{g(z)}{z g^{\prime}(z)}\right\}>\beta, \quad z \in \mathbb{D}
$$

for some $\beta, 0<\beta<1$. If

$$
\left|\arg \left\{\frac{f^{\prime}(z)}{g^{\prime}(z)}\right\}\right| \leq \pi-\tan ^{-1}\left\{\frac{2(1-\beta)|z|+1-|z|^{2}}{\beta\left(1-|z|^{2}\right)}\right\}, \quad z \in \mathbb{D},
$$

then we have

$$
\mathfrak{R e}\left\{\frac{f(z)}{g(z)}\right\}>0, \quad z \in \mathbb{D}
$$

Proof. If we put

$$
q(z)=\frac{f(z)}{g(z)}, \quad q(0)=1
$$

then it follows that

$$
f(z)=q(z) g(z), \quad f^{\prime}(z)=g^{\prime}(z) q(z)+q^{\prime}(z) g(z)
$$

and

$$
\frac{f^{\prime}(z)}{g^{\prime}(z)}=q(z)+q^{\prime}(z) \frac{g(z)}{g^{\prime}(z)}=q(z)+z q^{\prime}(z) \frac{g(z)}{z g^{\prime}(z)} .
$$

If there exists a point $z_{0} \in \mathbb{D}$, such that

$$
\mathfrak{R e}\{q(z)\}>0, \quad\left(|z|<\left|z_{0}\right|<1\right)
$$


and

$$
\mathfrak{R e}\left\{q\left(z_{0}\right)\right\}=0,
$$

then by (5), we have

$$
z_{0} q^{\prime}\left(z_{0}\right)=\mathfrak{R e}\left\{z_{0} q^{\prime}\left(z_{0}\right)\right\} \leq-\frac{1}{2}\left(1+\left|q\left(z_{0}\right)\right|^{2}\right)<0 .
$$

This shows that $z_{0} q^{\prime}\left(z_{0}\right)$ is a negative real number. Furthermore, by (4), we have

$$
\left|\frac{q\left(z_{0}\right)}{z_{0} q^{\prime}\left(z_{0}\right)}\right| \leq 1
$$

Then it follows that

$$
\frac{f^{\prime}\left(z_{0}\right)}{g^{\prime}\left(z_{0}\right)}=q\left(z_{0}\right)+z_{0} q^{\prime}\left(z_{0}\right) \frac{g\left(z_{0}\right)}{z_{0} g^{\prime}\left(z_{0}\right)}= \pm i a+z_{0} q^{\prime}\left(z_{0}\right) \frac{g\left(z_{0}\right)}{z_{0} g^{\prime}\left(z_{0}\right)}
$$

where $q\left(z_{0}\right)= \pm a i, a>0$ and (7), (10) give

$$
\begin{aligned}
\mathfrak{R e}\left\{\frac{f^{\prime}\left(z_{0}\right)}{g^{\prime}\left(z_{0}\right)}\right\} & =\mathfrak{R e}\left\{z_{0} q^{\prime}\left(z_{0}\right) \frac{g\left(z_{0}\right)}{z_{0} g^{\prime}\left(z_{0}\right)}\right\}=z_{0} q^{\prime}\left(z_{0}\right) \mathfrak{R e}\left\{\frac{g\left(z_{0}\right)}{z_{0} g^{\prime}\left(z_{0}\right)}\right\} \\
& <-\frac{\beta}{2}\left(1+a^{2}\right)<0 .
\end{aligned}
$$

Next, we have

$$
\mathfrak{I m}\left\{\frac{f^{\prime}\left(z_{0}\right)}{g^{\prime}\left(z_{0}\right)}\right\}=\mathfrak{I m}\left\{ \pm i a+z_{0} q^{\prime}\left(z_{0}\right) \frac{g\left(z_{0}\right)}{z_{0} g^{\prime}\left(z_{0}\right)}\right\}= \pm a+z_{0} q^{\prime}\left(z_{0}\right) \mathfrak{I m}\left\{\frac{g\left(z_{0}\right)}{z_{0} g^{\prime}\left(z_{0}\right)}\right\} .
$$

We will consider the four cases:

(i) $\arg \left\{q\left(z_{0}\right)\right\}=\pi / 2$ (i.e., $\left.q\left(z_{0}\right)=i a, a>0\right)$ and $\mathfrak{I m}\left\{\frac{f^{\prime}\left(z_{0}\right)}{g^{\prime}\left(z_{0}\right)}\right\} \geq 0$,

(ii) $\arg \left\{q\left(z_{0}\right)\right\}=\pi / 2$ (i.e., $\left.q\left(z_{0}\right)=i a, a>0\right)$ and $\mathfrak{I m}\left\{\frac{f^{\prime}\left(z_{0}\right)}{g^{\prime}\left(z_{0}\right)}\right\}<0$,

(iii) $\arg \left\{q\left(z_{0}\right)\right\}=-\pi / 2$ (i.e., $q\left(z_{0}\right)=-i a, a>0$ ) and $\mathfrak{I m}\left\{\frac{f^{\prime}\left(z_{0}\right)}{\bar{g}^{\prime}\left(z_{0}\right)}\right\} \geq 0$,

(iv) $\arg \left\{q\left(z_{0}\right)\right\}=-\pi / 2$ (i.e., $q\left(z_{0}\right)=-i a, a>0$ ) and $\mathfrak{I m}\left\{\frac{f^{\prime}\left(z_{0}\right)}{g^{\prime}\left(z_{0}\right)}\right\}<0$.

Let us put

$$
G(z)=\frac{p g(z)}{z g^{\prime}(z)}, \quad G(0)=1 .
$$

Then from the hypothesis, we have

$$
\frac{G(z)-\beta}{1-\beta} \prec \frac{1+z}{1-z}, \quad z \in \mathbb{D},
$$

and so we have

$$
G(z) \prec \beta+(1-\beta) \frac{1+z}{1-z}, \quad z \in \mathbb{D},
$$

and so

$$
|\mathfrak{I m}\{G(z)\}|=\left|\mathfrak{I m} \frac{p g(z)}{z g^{\prime}(z)}\right| \leq(1-\beta) \frac{2|z|}{1-|z|^{2}}, \quad z \in \mathbb{D} .
$$

In the case (i) we have $\arg \left\{q\left(z_{0}\right)\right\}=\pi / 2, q\left(z_{0}\right)=i a, a>0$ and

$$
\mathfrak{I m}\left\{\frac{f^{\prime}\left(z_{0}\right)}{g^{\prime}\left(z_{0}\right)}\right\}=\left|q\left(z_{0}\right)\right|+z_{0} q^{\prime}\left(z_{0}\right)\left(\mathfrak{I m}\left\{\frac{g\left(z_{0}\right)}{z_{0} g^{\prime}\left(z_{0}\right)}\right\}\right) \geq 0 .
$$


Therefore, we have

$$
\begin{aligned}
\arg \left\{\frac{f^{\prime}\left(z_{0}\right)}{g^{\prime}\left(z_{0}\right)}\right\} & =\arg \left[z_{0} q^{\prime}\left(z_{0}\right)\left(\mathfrak{R e} \frac{g\left(z_{0}\right)}{z_{0} g^{\prime}\left(z_{0}\right)}\right)+i\left\{\left|q\left(z_{0}\right)\right|+z_{0} q^{\prime}\left(z_{0}\right)\left(\mathfrak{I m} \frac{g\left(z_{0}\right)}{z_{0} g^{\prime}\left(z_{0}\right)}\right)\right\}\right] \\
& =\pi-\tan ^{-1}\left\{\frac{\left|q\left(z_{0}\right)\right|+z_{0} q^{\prime}\left(z_{0}\right)\left(\mathfrak{I m} \frac{g\left(z_{0}\right)}{z_{0} g^{\prime}\left(z_{0}\right)}\right)}{-z_{0} q^{\prime}\left(z_{0}\right)\left(\mathfrak{R e} \frac{g\left(z_{0}\right)}{z_{0} g^{\prime}\left(z_{0}\right)}\right)}\right\} \\
& >\pi-\tan ^{-1}\left\{\frac{\left|q\left(z_{0}\right)\right|+z_{0} q^{\prime}\left(z_{0}\right)\left(\mathfrak{I m} \frac{g\left(z_{0}\right)}{z_{0} g^{\prime}\left(z_{0}\right)}\right)}{-\beta z_{0} q^{\prime}\left(z_{0}\right)}\right\} \\
& =\pi-\tan ^{-1}\left\{-\frac{\left|q\left(z_{0}\right)\right|}{\beta z_{0} q^{\prime}\left(z_{0}\right)}-\frac{\mathfrak{I m} \frac{g\left(z_{0}\right)}{z_{0 g^{\prime}\left(z_{0}\right)}}}{\beta}\right\} \\
& \geq \pi-\tan ^{-1}\left\{\left|\frac{q\left(z_{0}\right)}{\beta z_{0} q^{\prime}\left(z_{0}\right)}\right|+\left|\frac{\mathfrak{I m} \frac{g\left(z_{0}\right)}{z_{0 g^{\prime}}\left(z_{0}\right)}}{\beta}\right|\right\} .
\end{aligned}
$$

Then, by (11) and (12), we have

$$
\begin{aligned}
\arg \left\{\frac{f^{\prime}\left(z_{0}\right)}{g^{\prime}\left(z_{0}\right)}\right\} & >\pi-\tan ^{-1}\left\{\frac{1}{\beta}+\frac{2(1-\beta)\left|z_{0}\right|}{\left(1-\left|z_{0}\right|^{2}\right) \beta}\right\} \\
& =\pi-\tan ^{-1}\left[\frac{1}{\beta\left(1-\left|z_{0}\right|^{2}\right)}\left\{2(1-\beta)\left|z_{0}\right|+1-\left|z_{0}\right|^{2}\right\}\right] .
\end{aligned}
$$

This contradicts hypothesis (8). In the case (ii) when $\arg \left\{q\left(z_{0}\right)\right\}=\pi / 2, q\left(z_{0}\right)=i a, a>0$, and

$$
\arg \left\{\frac{f^{\prime}\left(z_{0}\right)}{g^{\prime}\left(z_{0}\right)}\right\}=q\left(z_{0}\right)+z_{0} q^{\prime}\left(z_{0}\right)\left(\mathfrak{I m}\left\{\frac{g\left(z_{0}\right)}{z_{0} g^{\prime}\left(z_{0}\right)}\right\}\right)<0
$$

applying the same method as the above, we have

$$
\arg \left\{\frac{f^{\prime}\left(z_{0}\right)}{g^{\prime}\left(z_{0}\right)}\right\}<-\pi+\tan ^{-1}\left[\frac{1}{\beta\left(1-\left|z_{0}\right|^{2}\right)}\left\{2(1-\beta)\left|z_{0}\right|+1-\left|z_{0}\right|^{2}\right\}\right] .
$$

This is also a contradiction. In the case (iii) when $\arg \left\{q\left(z_{0}\right)\right\}=-\pi / 2, q\left(z_{0}\right)=-i a, a>0$, and

$$
q\left(z_{0}\right)+z_{0} q^{\prime}\left(z_{0}\right)\left(\mathfrak{I m}\left\{\frac{g\left(z_{0}\right)}{z_{0} g^{\prime}\left(z_{0}\right)}\right\}\right)>0
$$

and in the case (iv) when $\arg \left\{q\left(z_{0}\right)\right\}=-\pi / 2, q\left(z_{0}\right)=-i a, a>0$, and

$$
q\left(z_{0}\right)+z_{0} q^{\prime}\left(z_{0}\right)\left(\mathfrak{I m}\left\{\frac{g\left(z_{0}\right)}{z_{0} g^{\prime}\left(z_{0}\right)}\right\}\right)<0
$$

applying the same method as in the proof of case (i) gives

$$
\left|\arg \left\{\frac{f^{\prime}\left(z_{0}\right)}{g^{\prime}\left(z_{0}\right)}\right\}\right|>\pi-\tan ^{-1}\left[\frac{1}{\beta\left(1-\left|z_{0}\right|^{2}\right)}\left\{2(1-\beta)\left|z_{0}\right|+1-\left|z_{0}\right|^{2}\right\}\right] .
$$

This is a contradiction. This completes the proof.

Inequalities (9) and (2) show that the assumptions of Theorem 2 are sufficient for

$$
\int_{0}^{z} \frac{f(\zeta)}{g(\zeta)} \mathrm{d} \zeta
$$


to be univalent in $\mathbb{D}$.

Theorem 3. Let $F, G \in \mathcal{A}_{p}$. Assume that there exist a positive integer $k, 2 \leq k \leq p$ and a real $\beta, 0<\beta<1$, for which

$$
\left|\arg \left\{\frac{F^{(k)}(z)}{G^{(k)}(z)}\right\}\right|<\pi-\tan ^{-1}\left\{\frac{2(1-\beta)|z|+1-|z|^{2}}{\beta\left(1-|z|^{2}\right)}\right\}, \quad z \in \mathbb{D},
$$

where $G$ satisfies

$$
\mathfrak{R e}\left\{\frac{G^{(k-1)}(z)}{z G^{(k)}(z)}\right\}>\beta, \quad z \in \mathbb{D}
$$

Then

$$
\forall n \in\{1, \ldots, k-1\}: \quad \mathfrak{R e}\left\{\frac{F^{(n)}(z)}{G^{(n)}(z)}\right\}>0, \quad z \in \mathbb{D} .
$$

and $F \in \mathcal{K}_{p}, F$ is at most p-valent in $\mathbb{D}$.

Proof. If we put $f=F^{(k-1)}$ and $g=G^{(k-1)}$ in Theorem 2 we immediately obtain

$$
\mathfrak{R e}\left\{\frac{F^{(k-1)}(z)}{G^{(k-1)}(z)}\right\}>0, \quad z \in \mathbb{D} .
$$

Then, by Lemma 1, we obtain (14). For $n=1$ the condition (14) is of the form

$$
\mathfrak{R e}\left\{\frac{F^{\prime}(z)}{G^{\prime}(z)}\right\}>0, \quad z \in \mathbb{D},
$$

where $G$ satisfies (13). Therefore, by Lemma 1 we have also

$$
\mathfrak{R e}\left\{\frac{z G^{\prime}(z)}{G(z)}\right\}>0, \quad z \in \mathbb{D}
$$

which by (3) implies $F \in \mathcal{K}_{p}$. By Theorem $1, F$ is at most $p$-valent in $\mathbb{D}$.

Theorem 4. Assume that $f \in \mathcal{A}_{p}, 2 \leq p$, and that there exists a positive integer $k, 2 \leq k \leq p$ for which

$$
\mathfrak{R e}\left\{\frac{z f^{(k)}(z)}{f^{(k-1)}(z)}\right\}>-1, \quad z \in \mathbb{D},
$$

then we have

$$
\mathfrak{R e}\left\{\frac{z f^{\prime}(z)}{f(z)}\right\}>0, \quad z \in \mathbb{D},
$$

or $f$ is $p$-valent starlike in $\mathbb{D}$.

Proof. Let us put

$$
q_{1}(z)=\frac{1}{p-k+2} \frac{z f^{(k-1)}(z)}{f^{(k-2)}(z)}, \quad q_{1}(0)=1 .
$$

By (16) we have

$$
\frac{z q_{1}^{\prime}(z)}{q_{1}(z)}=1+\frac{z f^{(k)}(z)}{f^{(k-1)}(z)}-\frac{z f^{(k-1)}(z)}{f^{(k-2)}(z)}
$$

and so

$$
1+\frac{z f^{(k)}(z)}{f^{(k-1)}(z)}=\frac{z q_{1}^{\prime}(z)}{q_{1}(z)}+(p-k+2) q_{1}(z)
$$


By the hypothesis, we have

$$
1+\mathfrak{R e}\left\{\frac{z f^{(k)}(z)}{f^{(k-1)}(z)}\right\}=\mathfrak{R e}\left\{\frac{z q_{1}^{\prime}(z)}{q_{1}(z)}+(p-k+2) q_{1}(z)\right\}>0, \quad z \in \mathbb{D} .
$$

If there exists a point $z_{1} \in \mathbb{D}$, such that

$$
\mathfrak{R e}\left\{q_{1}(z)\right\}>0, \quad\left(|z|<\left|z_{1}\right|<1\right)
$$

and

$$
\mathfrak{R e}\left\{q_{1}\left(z_{1}\right)\right\}=0,
$$

then by Lemma 2, we have

$$
\mathfrak{R e}\left\{\frac{z_{1} q_{1}^{\prime}\left(z_{1}\right)}{q_{1}\left(z_{1}\right)}\right\}=0, \quad \frac{z_{1} q_{1}^{\prime}\left(z_{1}\right)}{q_{1}\left(z_{1}\right)}=i k_{1}
$$

for some real $k_{1},\left|k_{1}\right| \geq 1$. This gives

$$
1+\mathfrak{R e}\left\{\frac{z f^{(k)}\left(z_{1}\right)}{f^{(k-1)}\left(z_{1}\right)}\right\}=\mathfrak{R e}\left\{\frac{z_{1} q_{1}^{\prime}\left(z_{1}\right)}{q_{1}\left(z_{1}\right)}+(p-k+2) q_{1}\left(z_{1}\right)\right\}=0 .
$$

It is contrary to inequality (17) and therefore, we have

$$
\mathfrak{R e}\left\{\frac{z f^{(k-1)}(z)}{f^{(k-2)}(z)}\right\}>\mathfrak{R e}\left\{\frac{1}{p-k+2} \frac{z f^{(k-1)}(z)}{f^{(k-2)}(z)}\right\}=\mathfrak{R e}\left\{q_{1}(z)\right\}>0, \quad z \in \mathbb{D} .
$$

Next, let us put

$$
q_{2}(z)=\frac{1}{p-k+3} \frac{z f^{(k-2)}(z)}{f^{(k-3)}(z)}, \quad q_{2}(0)=1,
$$

then it follows that

$$
\frac{z q_{2}^{\prime}(z)}{q_{2}(z)}=1+\frac{z f^{(k-1)}(z)}{f^{(k-2)}(z)}-\frac{z f^{(k-2)}(z)}{f^{(k-3)}(z)}
$$

and so

$$
1+\frac{z f^{(k-1)}(z)}{f^{(k-2)}(z)}=\frac{z q_{2}^{\prime}(z)}{q_{2}(z)}+(p-k+3) q_{2}(z) .
$$

By (18) and (19), we have

$$
1+\mathfrak{R e}\left\{\frac{z f^{(k-1)}(z)}{f^{(k-2)}(z)}\right\}=\mathfrak{R e}\left\{\frac{z q_{2}^{\prime}(z)}{q_{2}(z)}+(p-k+3) q_{2}(z)\right\}>0, \quad z \in \mathbb{D} .
$$

If there exists a point $z_{2} \in \mathbb{D}$, such that

$$
\mathfrak{R e}\left\{q_{2}(z)\right\}>0, \quad\left(|z|<\left|z_{2}\right|<1\right)
$$

and

$$
\mathfrak{R e}\left\{q_{2}\left(z_{2}\right)\right\}=0,
$$

then by Lemma 2, we have

$$
\mathfrak{R e}\left\{\frac{z_{2} q_{2}^{\prime}\left(z_{2}\right)}{q_{2}\left(z_{2}\right)}\right\}=0, \quad \frac{z_{2} q_{2}^{\prime}\left(z_{2}\right)}{q_{2}\left(z_{2}\right)}=i k_{2}
$$


for some real $k_{2},\left|k_{2}\right| \geq 1$. Then, we have

$$
1+\mathfrak{R e}\left\{\frac{z_{2} f^{(k-1)}\left(z_{2}\right)}{f^{(k-2)}\left(z_{2}\right)}\right\}=\mathfrak{R e}\left\{\frac{z_{2} q_{2}^{\prime}\left(z_{2}\right)}{q_{2}\left(z_{2}\right)}+(p-k+3) q_{2}\left(z_{2}\right)\right\}=0 .
$$

It is contrary to (20) and therefore, we have

$$
\mathfrak{R e}\left\{\frac{z f^{(k-2)}(z)}{f^{(k-3)}(z)}\right\}=\mathfrak{R e}\left\{(p-k+3) q_{2}(z)\right\}>0, \quad z \in \mathbb{D} .
$$

Applying the same method many times in succession we are able to obtain

$$
\mathfrak{R e}\left\{\frac{z f^{(k-3)}(z)}{f^{(k-4)}(z)}\right\}>0, \mathfrak{R e}\left\{\frac{z f^{(k-4)}(z)}{f^{(k-5)}(z)}\right\}>0 \ldots, \mathfrak{R e}\left\{\frac{z f^{\prime}(z)}{f(z)}\right\}>0, \quad z \in \mathbb{D}
$$

This shows that $f$ is $p$-valent starlike in $\mathbb{D}$.

For some related conditions for starlikeness we refer to our papers $[9,10]$.

Author Contributions: All authors have equal contributions.

Funding: This research received no external funding.

Conflicts of Interest: The authors declare no conflict of interest.

\section{References}

1. Noshiro, K. On the theory of schlicht functions. J. Fac. Sci. Hokkaido Univ. Jap. 1934, 2, 129-135. [CrossRef]

2. Warschawski, S. On the higher derivatives at the boundary in conformal mapping. Trans. Am. Math. Soc. 1935, 38, 310-340. [CrossRef]

3. Ozaki, S. On the theory of multivalent functions. Sci. Rep. Tokyo Bunrika Daigaku Sect. A 1935, 2, 167-188.

4. Nunokawa, M. A note on multivalent functions. Tsukuba J. Math. 1989, 13, 453-455. [CrossRef]

5. Umezawa, T. Multivalently close-to-convex functions. Proc. Am. Math. Soc. 1957, 8, 869-874. [CrossRef]

6. Nunokawa, M. On the theory of multivalent functions. Tsukuba J. Math. 1987, 11, 273-286. [CrossRef]

7. Nunokawa, M. On Properties of Non-Carathéodory Functions. Proc. Jpn. Acad. Ser. A 1992, 68, 152-153. [CrossRef]

8. Miller, S.S.; Mocanu, P.T. Differential Subordinations, Theory and Applications, Series of Monographs and Textbooks in Pure and Applied Mathematics; Marcel Dekker Inc.: New York, NY, USA; Basel, Switzerland, 2000; Volume 225.

9. Sokół, J. On a condition for alpha-starlikeness. J. Math. Anal. Appl. 2009, 352, 696-701. [CrossRef]

10. Nunokawa, M.; Sokół, J. On some sufficient condition for starlikeness. J. Ineq. Appl. 2012, 2012, 282.

(C) 2019 by the authors. Licensee MDPI, Basel, Switzerland. This article is an open access article distributed under the terms and conditions of the Creative Commons Attribution (CC BY) license (http:/ / creativecommons.org/licenses/by/4.0/). 